\section{Novel SCN5A mutation associated with idiopathic ventricular fibrillation due to subclinical Brugada syndrome}

\author{
Juan Jiménez-Jáimez, \\ Miguel Álvarez-López, \\ Luis Tercedor-Sánchez, Pablo Santiago, \\ Maria Algarra, Rocio Peñas, \\ Francisca Valverde, \\ Rafael Melgares-Moreno \\ Cardiology Department, Virgen de las \\ Nieves Universitary Hospital, Granada, \\ Spain
}

\section{Abstract}

Idiopathic ventricular fibrillation can be caused by subclinical channelopathies such as Brugada syndrome. Our objective is to study the clinical behaviour of a new SCN5A mutation found in a woman with idiopathic ventricular fibrillation. A 53-year-old woman presented with multiple episodes of ventricular fibrillation, a structurally normal heart and normal baseline electrocardiogram. Genetic testing included KCNQ1, KCNH2, SCN5A, KCNE1, KCNE2 and KCNJ2 and identified a mutation in SCN5A (D1816fs/g98747-98748insT). We studied 15 immediate family members by means of electrocardiogram, echocardiogram, flecainide challenge test and genetic study. Eight subjects had the mutation. The flecainide challenge test was positive for Brugada syndrome in two subjects in the case group and none in the control group. The PR and QRS intervals on the baseline electrocardiogram were longer in the case group. The left atrial volume indexed to body surface was higher in the case group, likely due to the fact that two patients with the mutation had atrial fibrillation and none had it in the control group. The D1816fs/g98747-98748insT mutation in SCN5A may be associated with idiopathic ventricular fibrillation and Brugada syndrome with a broad phenotypic spectrum and incomplete penetrance. Genetic testing may be useful to identify the etiology of idiopathic ventricular fibrillation in patients with a negative thorough clinical evaluation.

\section{Introduction}

Ventricular fibrillation (VF) is a lethal arrhythmia that leads to a dramatic reduction in cardiac output and causes cardiac arrest and sudden death in several minutes if cardiopulmonary resuscitation manoeuvres are not begun quickly. It usually occurs as a result of structural heart conditions such as ischaemic heart disease and cardiomyopathy. ${ }^{1,2}$ After ruling out structural abnormalities, the most prevalent cause of VF are genetic channelopathies, including long QT syndrome (LQTS), Brugada syndrome (BS) and catecholaminergic polymorphic ventricular tachycardia $^{3-5}$ If all diagnostic tests are negative, it is classified as idiopathic ventricular fibrillation (IVF). This condition is uncommon because diagnostic studies have an increasingly wide scope and provide a confirmed diagnosis in most cases. ${ }^{6}$

Subclinical channelopathies are one possible cause of IVF. Primary cardiac ion-channel diseases can be difficult to diagnose because of their limited phenotypic expression in surface electrocardiograms (ECG) and in drug challenge tests due to their incomplete genetic penetrance. ${ }^{7}$ In these cases, genetic studies are of growing importance. ${ }^{6}$ The increasing availability of genetic studies has led to a larger number of subclinical channelopathy diagnoses that initially present as IVF. ${ }^{8}$ However, genetic findings must be interpreted correctly, since most mutations detected have not been described and many are not clinically relevant. ${ }^{9}$

We conducted an exhaustive clinical study of a novel mutation in SCN5A (D1816fs/g 98747-98748insT) in a Spanish family in which the index case presented as IVF. We have not found previous descriptions of this variant and there are no functional studies in a cellular context. Previous studies have involved mutations in nearby locations on the protein both in LQTS and also in BS,,$^{10,11}$ but there is no evidence of this mutation causing cardiac ion-channel diseases or IVF.

Diagnostic possibilities include LQTS type 3 or BS, or no pathogenic implications in the case of this mutation. Our main objective was to study the clinical pathogenicity and association of this mutation with possible subclinical channelopathy in the form of LQTS type 3 or BS.

\section{Materials and Methods}

\section{Description of the index case}

A 53-year-old woman without personal or family history of interest presented an episode of VF that was successfully treated. The initial study involved an ECG (Figure 1), echocardiogram, cardiovascular magnetic resonance and coronary angiography. The QTc interval calculated by Bazzet's formula ${ }^{12}$ was 412 milliseconds and there were no signs suggesting Brugada pattern in right precordial leads. The left ventricular ejection fraction was $60 \%$, with no evidence of hypertrophy or chamber dilatation. Coronary arteries showed no significant atherosclerotic lesions.
Correspondence: Juan Jiménez Jáimez, Cardiology Department, Hospital Universitario Virgen de las Nieves. Avda. de las Fuerzas Armadas 2, 18014. Granada, Spain

Tel. +34.667.242.021.

E-mail: jiménez-jaimez@gmail.com

Key words: idiopathic ventricular fibrillation, cardiac arrest, gene mutation, Brugada syndrome.

Contributions: all the authors participated actively in this work. JJJ collected all the data and wrote the manuscript.

Conflicts of interests: the authors declare no conflicts of interests.

Received for publication: 26 0ctober 2011 Revision received: 29 November 2011.

Accepted for publication: 14 December 2011.

This work is licensed under a Creative Commons Attribution NonCommercial 3.0 License (CC BYNC 3.0).

(OCopyright J. Jiménez-Jáimez et al., 2012

Licensee PAGEPress, Italy

Cardiogenetics 2012; 2:e1

doi:10.4081/cardiogenetics.2012.e1

A drug challenge test was performed with an initial bolus of $0.10 \mu \mathrm{g} / \mathrm{kg}$ epinephrine, followed by continuous infusion at $0.10 \mu \mathrm{g} / \mathrm{kg} /$ min. for $10 \mathrm{~min}$., performing a twelve-lead ECG. The test was negative for LQTS according to current criteria. ${ }^{13}$ After a washout period, we performed a flecainide challenge test giving 2 $\mathrm{mg} / \mathrm{kg}$ in a continuous infusion for $10 \mathrm{~min},{ }^{14}$ with a baseline ECG and further recordings every $2 \mathrm{~min}$. This was negative for BS.

An electrophysiological study was performed in view of recurrent episodes of VF triggered by ventricular extrasystoles on the upper-left axis and short accompanying interval (Figure 2A), which was resistant to continuous infusion of beta blockers, amiodarone, procainamide and isoprenaline. Along the mid-apical septum of the right ventricle, Purkinje potentials were recorded at a level of the distal insertion of the right branch. These potentials preceded the onset of the QRS of the VF-inducing ventricular extrasystole by $45 \mathrm{~ms}$, and the VF was self-limiting on one occasion. Radiofrequency applications eliminated the ventricular extrasystoles and VF episodes (Figure 2B). On day 7, a single-chamber automatic defibrillator was implanted, and during 18 months of follow-up, the device had not recorded any ventricular arrhythmias and the ECG remains normal.

In view of all these data, the final diagnosis was IVF. Considering how useful genetic testing has been in previous studies for LQTS and BS in patients with IVF, ${ }^{6,8}$ we performed genetic sequencing of the genes $K C N Q 1, K C N H 2$, 
SCN5A, KCNE1, KCNE2 and KCNJ2. Analysis included the entire genes. In SCN5A we identified a heterozygous insertion-type mutation (D1816fs/g98747-98748insT) in exon 28, affecting the $\mathrm{C}$-terminal domain (Figure 3 ). There were no mutations in the rest of the genes.

\section{Study population}

16 subjects were included (15 immediate family members and the index case). The family tree is shown in Figure 4. A detailed history was taken on all subjects, with emphasis on past syncopes. The study protocol for these subjects consisted of an ECG, echocardiography, flecainide challenge test and genetic study focused on the SCN5A mutation found in the proband. All subjects signed an informed consent in order to be included in the study. All the procedures performed were approved by the Ethics Committee at our hospital.

One of the immediate family members was excluded from the electrocardiographic, echocardiographic and drug challenge tests since she had Tetralogy of Fallot, having undergone surgery in childhood for this condition, which altered the baseline values of these studies.

\section{Electrocardiogram}

The surface ECGs at 25 and $50 \mathrm{~mm} / \mathrm{s}$ were evaluated by two cardiologists specialising in electrophysiology. The PR, QRS and QTc intervals were analysed using Bazzet's formula. ${ }^{12}$ The presence of type 1 Brugada pattern was defined as persistent ST segment elevation in right precordial leads with typical morphology. The presence of atrial fibrillation on ECG was confirmed with a second ECG three months later.

\section{Measurement of echocardiographic parameters}

Echocardiographic studies were performed with the Acuson X300 System (Siemens ${ }^{\circledR}$ ). All

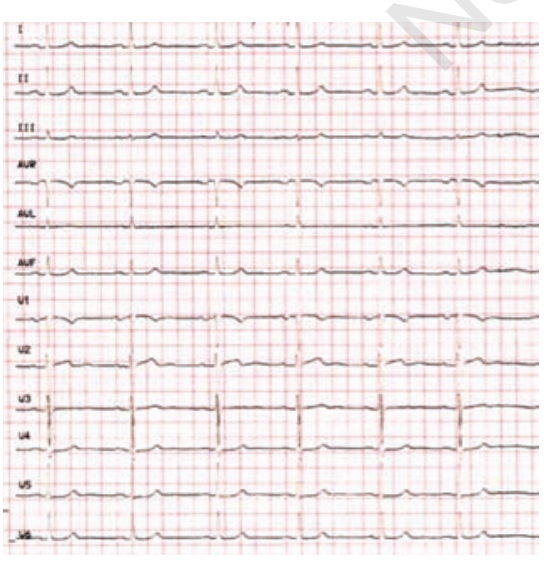

Figure 1. Baseline electrocardiogram of the index case showing sinus rhythm at $\mathbf{5 5}$ beats per min, normal PR, QRS and QTc intervals and absence of Brugada pattern findings. measurements were taken following the guidelines of the American Society of Echocardiography. ${ }^{15}$

The left ventricular ejection fraction was calculated using the modified Simpson method. The left atrial anteroposterior diameter was measured on the parasternal long axis at ventricular end-systole. The left atrial lateral diameter was measured in the apical fourchamber view, also at ventricular end-systole. The left atrial volume was calculated by biplane measurements in the apical two- and fourchamber views at ventricular end-systole. The endocardial border of the left atrium was traced, excluding pulmonary veins and the left atrial appendage. The left atrial volume was later calculated using the modified Simpson method, indexing it to body surface area.

Data were obtained on peak velocity of early and late diastolic mitral filling ( $\mathrm{E}$ and $\mathrm{A}$ waves respectively).

\section{Flecainide challenge test}

For the drug challenge test we administered flecainide intravenously peripherally, and performed continuous electrocardio-

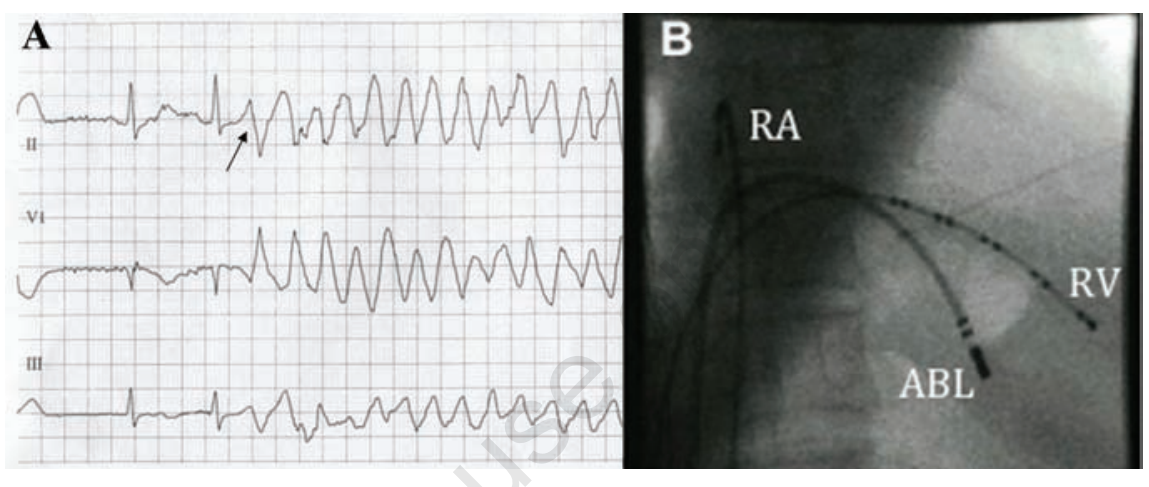

Figure 2. A) Electrocardiogram recording on admission, with ventricular fibrillation episode triggered by ventricular extrasystoles (arrow). B) Fluoroscopy-guided radiofrequency ablation of the ventricular focus triggering the VF episodes. RA, right atrium; RV, right ventricle; $A B L$, ablation catheter.

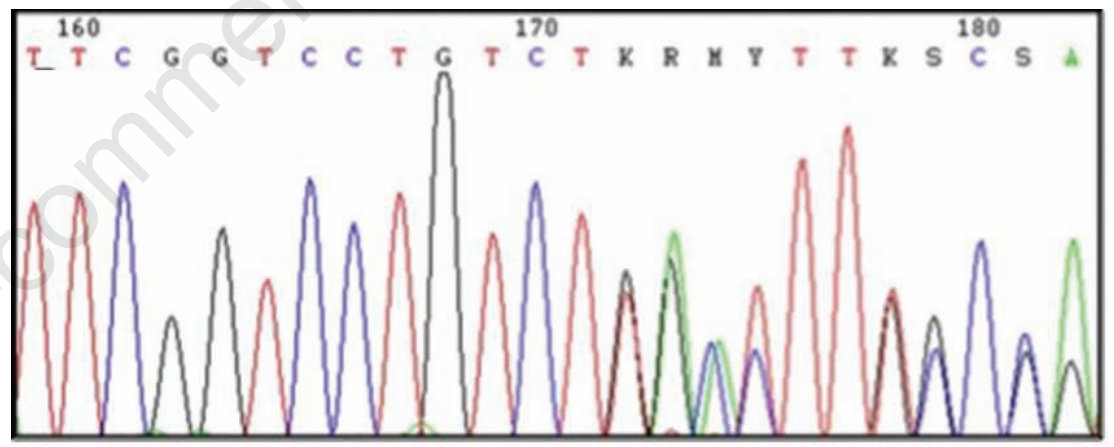

Figure 3. Heterozygous insertion mutation D1816fs/g98747-98748insT in exon 28 of SCN5A.

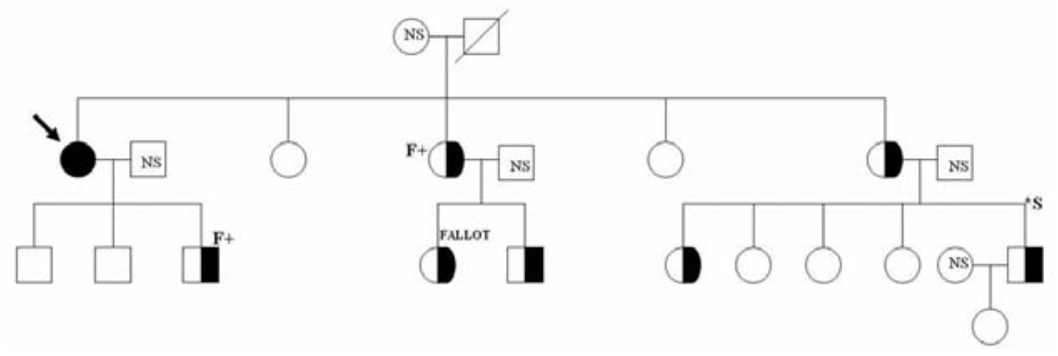

Figure 4. Family tree. The proband is shown as a black circle (arrow). Asymptomatic carriers of the mutation (cases) are shown as black and white circles and squares (circles=female; squares=male). Family members who did not have the mutation (controls) are shown as white circles and squares. ${ }^{*} S$ marks the case with syncope. F+ shows family members with a positive flecainide test. "Fallot" marks the case excluded from the electrocardiogram and imaging study because of Tetralogy of Fallot. NS, Not studied. 
graphic monitoring. Two $\mathrm{mg} / \mathrm{kg}$ flecainide was infused as a continuous infusion for 10 min., performing a baseline ECG and repeating a recording every $2 \mathrm{~min}$. The right precordial leads were located in the usual position (fourth intercostal space) and also one intercostal space higher up. ST segment behaviour was monitored from V1-V3, looking for any signs of BS. The test was classified as positive, negative or indeterminate according to previously published standards. It was considered positive if an ST elevation $>1 \mathrm{~mm}$ was observed in right precordial leads with Brugada pattern typical morphology. ${ }^{14}$

\section{Genetic study}

We took a blood sample for direct sequencing and DNA analysis of immediate family members in order to identify the presence of the SCN5A mutation that had been found in the index case (Figure 3).

\section{Statistical analysis}

The study subjects $(n=16)$ were divided into two groups for statistical comparison. One group consisted of patients with the SCN $5 A$ mutation (case group, $n=8$ ) and the other group was composed of immediate family members without the $S C N 5 A$ mutation (control group, $n=8$ ). A statistical comparison was performed in all cases using the aforementioned electrocardiographic, echocardiographic and drug challenge test data as dependent variables.

Nonparametric tests were used to calculate the $P$ : the Mann Whitney U test to compare the mean and standard deviation and Fisher's exact test and the Chi-square test to compare proportions. A P value $<0.05$ was considered as statistically significant. To study concordance between the two observers in the analysis of electrocardiographic parameters we used a nonparametric correlation test (Spearman's Rho coefficient). All calculations were performed with SPSS 17.0 (Chicago, USA).

The authors of this manuscript have certified that they comply with the Principles of Ethical Publishing in the International Journal of Cardiology. ${ }^{16}$

\section{Results}

Eight immediate family members had the mutation in SCN5A found in the index case and eight did not (Figure 4). There were no statistically significant differences between groups in terms of age or sex. One subject in the group with the SCN5A mutation had suffered an episode of VF (the proband) and another subject had had a exertional syncopal episode of uncertain origin ten years before the study and is under follow up; the rest were asymptomatic. All subjects in the control group were asymptomatic. The result of the statistical comparison between the two groups analysing electrocardiographic, echocardiographic and flecainide test parameters is shown in Table 1.

\section{Electrocardiogram abnormalities}

There was very high correlation between the two observers' measurements of the PR and QTc intervals and high correlation in their evaluation of the QRS interval (Table 2).

No patients in the case group or control group showed the Brugada pattern on their baseline ECG. The QTc interval was similar in both groups without statistically significant differences.

The value of the PR interval in milliseconds was significantly higher in subjects with the SCN5A mutation, compared with the controls (196.7 \pm 27.6 vs. $150 \pm 28.9$ milliseconds, respectively). The QRS interval was also statistically significantly higher in the cases compared with the controls $(112.8 \pm 16.6$ vs $90.8 \pm 12.4$

milliseconds, respectively). Atrial fibrillation was present in two cases and in none of the controls (Table 1).

\section{Alterations in echocardiographic parameters}

The left atrial volume indexed to body surface area was significantly higher in the SCN5A mutation cases compared with controls, athough this included the two patients that had atrial fibrillation. The mean volume indexed to body surface area in the cases was $40.92 \pm 8.9 \mathrm{~mL} / \mathrm{m}^{2}$ and in controls it was $32.4 \pm 5.8 \mathrm{~mL} / \mathrm{m}^{2}$. The volume was bigger in the two subjects who presented atrial fibrillation compared with the others. These two cases had the SCN5A mutation and one of them was the index case that had had an episode of VF.

The left atrial anteroposterior diameter measured on the parasternal long axis was greater in the cases than in the controls. However, the difference in lateral diameter was not of statistical significance.

The other echocardiographic parameters did

Table 1. Statistical comparison between the two groups analysing electrocardiographic, echocardiographic and flecainide test parameters.

\begin{tabular}{|c|c|c|c|}
\hline $0^{(D 1}$ & $\begin{array}{l}\text { SCN5A + } \\
\text { /g98747-98748insT) } \\
(\mathrm{n}=8)\end{array}$ & $\begin{array}{l}\text { SCN5A- } \\
(\mathrm{n}=8)\end{array}$ & $P$ \\
\hline $\begin{array}{l}\text { Clinical parameters } \\
\text { Age (years) } \\
\text { Men/Women } \\
\text { Syncope } \\
\text { VF }\end{array}$ & $\begin{array}{c}38 \pm 15 \\
3 / 5 \\
1(12.5 \%) \\
1(12.5 \%)\end{array}$ & $\begin{array}{l}31 \pm 19 \\
2 / 6 \\
0(0 \%) \\
0(0 \%)\end{array}$ & $\begin{array}{l}N S \\
N S \\
N S \\
N S\end{array}$ \\
\hline $\begin{array}{l}\text { ECG parameters } \\
\text { Spontaneous Brugada pattern (\%) } \\
\text { PR interval (ms) } \\
\text { QRS interval (ms) } \\
\text { QTc interval (ms) } \\
\text { Atrial fibrillation (\%) }\end{array}$ & $\begin{array}{l}0(0 \%) \\
196.7 \pm 27 \\
112.8 \pm 16 \\
393 \pm 25 \\
2(25 \%)\end{array}$ & $\begin{array}{l}0(0 \%) \\
150 \pm 29 \\
90.8 \pm 12 \\
399 \pm 22 \\
0(0 \%)\end{array}$ & $\begin{array}{l}N S \\
0.01 \\
0.04 \\
N S \\
N S\end{array}$ \\
\hline $\begin{array}{l}\text { Echocardiographic parameters } \\
\text { LA anteroposterior diameter (mm) } \\
\text { LA lateral diameter }(\mathrm{mm}) \\
\text { Peak E wave velocity }(\mathrm{cm} / \mathrm{s}) \\
\text { Peak A wave velocity }(\mathrm{cm} / \mathrm{s}) \\
\text { Left ventricular EF }(\%) \\
\text { Indexed LA volume }(\mathrm{mL} / \mathrm{m} 2)\end{array}$ & $\begin{array}{c}41.6 \pm 3.8 \\
43.1 \pm 6.5 \\
75 \pm 11 \\
50 \pm 20 \\
63 \% \\
40.9 \pm 8.9\end{array}$ & $\begin{array}{c}32.3 \pm 4.7 \\
39.8 \pm 5 \\
84 \pm 9 \\
61 \pm 19 \\
61 \% \\
32.4 \pm 5.8\end{array}$ & $\begin{array}{l}<0.05 \\
N S \\
N S \\
N S \\
N S \\
<0.05\end{array}$ \\
\hline Positive drug test for BS (\%) & $2(25 \%)$ & $0(0 \%)$ & $N S$ \\
\hline
\end{tabular}

LA, Left atrium; EF, Ejection fraction; BS, Brugada syndrome; VF, Ventricular fibrillation; NS, Not significant.

Table 2. Concordance between the two observers for electrocardiogram measurements expressed by Spearman's Rho correlation coefficient ( 0 : no concordance, 1: full concordance).

\begin{tabular}{llccc} 
& & Observer 1 & Observer 2 & Spearman's Rho coefficient \\
\multirow{2}{*}{ PR interval (ms) } & Cases & $196.7 \pm 25$ & $196.7 \pm 29$ & 0.947 \\
& Controls & $155 \pm 22$ & $145 \pm 30$ & \\
QRS interval (ms) & Cases & $116.5 \pm 23$ & $109.1 \pm 13$ & 0.744 \\
& Controls & $94.2 \pm 14$ & $87.5 \pm 10$ & \\
\hline \multirow{2}{*}{ QTc interval (ms) } & Cases & $390.2 \pm 26$ & $395.8 \pm 24$ & 0.912 \\
& Controls & $403 \pm 20$ & $395 \pm 22$ & \\
\hline
\end{tabular}


not differ between the groups, including left ventricular systolic function and diastolic transmitral flow velocities.

\section{Flecainide drug test}

Two cases with SCN5A mutation developed the typical Brugada pattern following intravenous infusion of flecainide. They were both asymptomatic and one of them had a QRS interval in the upper limit of the normal range in the baseline ECG (Figure 5). However, the index case, which showed a more aggressive clinical phenotype with VF, did not have any electrocardiographic changes following the drug challenge.

No subjects were positive for Brugada syndrome pattern in the control group. This result was not statistically significant (Table 1).

Flecainide test sensitivity and specificity fo the identification of the SCN5A mutation carriers was $29 \%$ and $100 \%$ respectively.

\section{Discussion}

A diagnosis of IVF means that it has been impossible to diagnose the underlying aetiology in a case of recovery from cardiac arrest. Recently, genetic studies have been found to play a part in the diagnostic protocol, detecting masked channelopathies or mutations that generate major distortions in proteins in cardiac ion channels, and which are predisposing factors for VF. Cardiac sodium channel alpha subunit mutations ( $\mathrm{Na} 1.5 \mathrm{~V}$ ) are a known cause for Brugada syndrome, being present in around $20 \%$ of patients. However, having a SNC5A mutation has been also related to Long QT syndrome, dilated cardiomyopathy and cardiac conduction system disease. So SCN5A mutations can lead to ventricular fibrillation and sudden death by different mechanisms, suggesting it plays an important role in cardiac electrical stability.

In this study we have provided clinical evidence of the causality of a previously undescribed mutation in SCN5A in BS, which presented as IVF. We identified the nucleotide insertion mutation D1816fs/g98747-98748insT, located in exon 28 , in the C-terminal region of the alpha subunit of the sodium channel, in a Spanish family affected by sudden cardiac death of unknown aetiology. This variant was not present in a sample of 200 healthy control subjects.

We have not found previous descriptions of this variant and there are no studies that analyse the behaviour of this variant in a cellular context. However, there is consensus that the first portion of the C-terminal region, which has a helical structure, plays a determinant role in stabilising the channel inactivation process, thus contributing to cardiac repo-

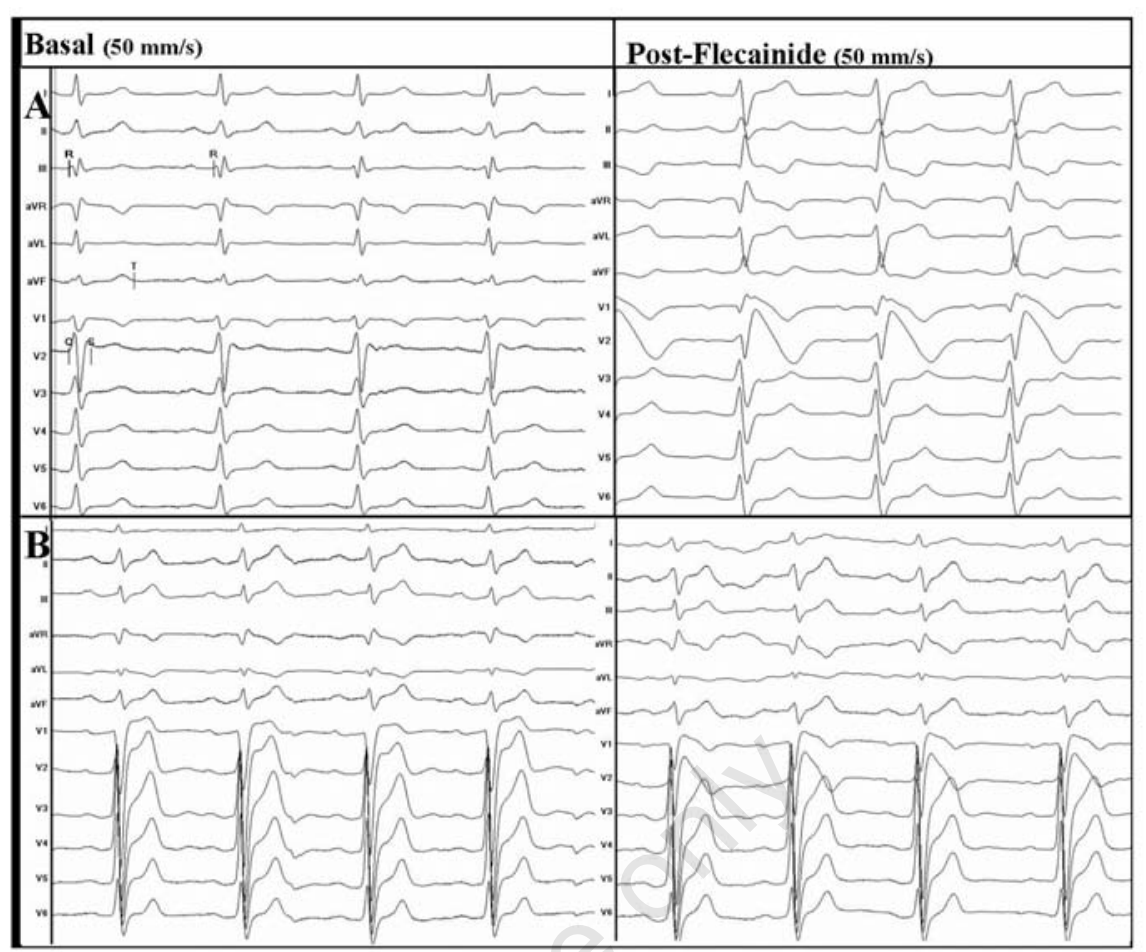

Figure 5. Positive flecainide challenge test for Brugada syndrome in two asymptomatic family members. The baseline electrocardiogram in B also shows a slightly widened QRS.

larisation. ${ }^{17}$ Previous studies have analysed the functional consequences of mutations in nearby regions in the protein on the physiology of cardiac action potential. Thus, Tan et al. ${ }^{11}$ described a family with carriers of the L1821fs mutation who developed different conduction disturbances, atrial fibrillation and ventricular tachycardia. Furthermore, Cormier et al. ${ }^{10}$ evaluated the functional changes associated with channel mutation at position 1885 , finding a tenfold associated increase in the percentage of channels that fail in the inactivation process, leading to functional gain and therefore potential QT interval prolongation.

The insertion-type mutation that we have described here changes the reading frame in a large number of nucleotides, generating a truncated protein. There is a high probability that the patient's IVF is caused by this mutation because it changes the amino acid sequence in an important region in the SCN $5 A$ ion channel, with a probable impact on channel physiology and action potential. A functional study in a cellular model could be helpful to support the pathogenicity of the mutation and to evaluate its functional effect. This is not always feasible, although, as our work suggests, familial cosegregation data and clinical studies are the main way to evaluate the pathogenicity of a novel mutation. In this case we used a clinical study protocol to test the Spanish family carrying the mutation in the Cterminal region of SCN5A. Our aims in doing so were to identify the possible association of this mutation with subclinical channelopathy and to analyse its clinical behaviour.

The diagnoses of LQTS and the BS are mainly based on clinical, electrocardiographic and drug challenge test findings. ${ }^{18}$ Some studies have associated BS with prolonged conduction intervals in surface ECGs, especially with reference to the QRS interval. ${ }^{19}$ Drug challenge tests with epinephrine and flecainide show low sensitivity but high specificity in detecting channelopathies, and so they provide a valuable diagnostic tool in the study of this novel mutation in $S C N 5 A .{ }^{7}$ Echocardiography might play a role in $S C N 5 A$ mutation carriers presenting with atrial fibrillation, particularly focusing on left atrial size and volume measurements, which have been previously studied in BS. $^{20}$

Our results suggest an association between this mutation and baseline and post-flecainide electrocardiographic. Although some of these results are not statistically significant there is a clear tendency towards the association between this mutation and the presence of the Brugada pattern after the flecainide infusion. These findings suggest that the D1816fs/g98747-98748insT mutation located in exon 28 of $S C N 5 A$ is pathogenic and is responsible for the development of $\mathrm{BS}$. Likewise, our work shows a tendency to the association of this mutation with atrial fibrillation, as previously reported. ${ }^{21}$ 


\section{Broad clinical spectrum and incomplete penetrance}

A wide range of phenotypic manifestations has been reported for BS, from asymptomatic patients, to syncope and ventricular fibrillation. Similarly, the electrocardiographic manifestations of spontaneous and post-flecainide Brugada pattern are not present in all cases. ${ }^{22}$ Our study confirms these findings in BS caused by the reported SCN5A mutation, with only one patient among the eight cases that were studied presenting VF, another with syncope and six being asymptomatic. Interestingly, none of them showed spontaneous Brugada pattern, and only two of them showed it following an intravenous flecainide infusion. Surprisingly, the index case who had suffered an episode of ventricular fibrillation did not have any alternation in her baseline or post-flecainide ECG, although she did have atrial fibrillation and left atrial enlargement. This is consistent with previous studies that suggest a negative prognostic value of atrial fibrillation and left atrial volume in BS. ${ }^{20}$

\section{Conclusions}

The heterozygous insertion mutation D1816fs/g98747-98748insT present in exon 28 of the C-terminal region of $S C N 5 A$, is likely associated with BS and IVF. It also seems to promote the development of atrial fibrillation. Our study describes a broad phenotypic spectrum with a majority of asymptomatic patients, as well as low clinical penetrance in view of the normal baseline and post-flecainide ECG found in most patients. This underlines the need for a complete family study with baseline ECG and flecainide challenge test in patients who have IVF.

\section{References}

1. Connolly SJ, Hallstrom AP, Cappato R, et al. Meta-analysis of the implantable cardioverter defibrillator secondary prevention trials: AVID, CASH and CIDS studies: Antiarrhythmics vs Implantable Defibrillator study; Cardiac Arrest Study Hamburg;
Canadian Implantable Defibrillator Study. Eur Heart J 2000;21:2071-8.

2. Behr E, Wood DA, Wright M, et al. Cardiological assessment of first-degree relatives in sudden arrhythmic death syndrome. Lancet 2003;362:1457-9.

3. Zareba W, Moss AJ, Schwartz PJ, et al. Influence of genotype on the clinical course of the long-QT syndrome: International Long-QT syndrome Registry Research Group. N Engl J Med 1998;339: 960-5.

4. Priori SG, Napolitano C, Tiso N, et al. Mutations in the cardiac ryanodine receptor gene (hRyR2) underlie catecholaminergic polymorphic ventricular tachycardia. Circulation 2001;16:196-200.

5. Priori SG, Napolitano C, Gasparini M, et al. Natural history of Brugada syndrome: insights for risk stratification and management. Circulation 2002;105:1342-7.

6. Krahn AD, Healey JS, Chauhan V, et al. Systematic assessment of patients with unexplained cardiac arrest: Cardiac Arrest Survivors With Preserved Ejection Fraction Registry (CASPER). Circulation 2009.28;120:278-85

7. Krahn AD, Gollob M, Yee R, et al. Diagnosis of unexplained cardiac arrest: role of adrenaline and procainamide infusion. Circulation 2005;112:2228-34.

8. Jiménez-Jáimez J, Tercedor-Sánchez L, Alvarez-López M, et al. Estudio genético en el Síndrome de QT largo en nuestro medio. Rev Esp Cardiol 2011;64:71-4.

9. Darbar D. Is it time to develop a "pathogenicity" score to distinguish long QT syndrome causing mutations from "background" genetic noise? Heart Rhythm 2009;6:1304-5.

10. Cormier JW, Rivolta I, Tateyama M, et al. Secondary structure of the human cardiac $\mathrm{Na}+$ channel $\mathrm{C}$ terminus: evidence for a role of helical structures in modulation of channel inactivation. J Biol Chem 2002;15; 277:9233-41.

11. Tan HL, Hofman N, van Langen IM, et al. Sudden unexplained death: heritability and diagnostic yield of cardiological and genetic examination in surviving relatives. Circulation 2005;112:207-13.

12. Bazett $H$. An analysis of the time-relations of electrocardiograms. Heart 1920;7:35370 .
13. Shimizu W, Noda T, Takaki H, et al. Epinephrine unmasks latent mutation carriers with LQT1 form of congenital longQT syndrome. J Am Coll Cardiol 2003;41: 633-42.

14. Brugada R, Brugada J, Antzelevitch C, et al. Sodium channel blockers identify risk for sudden death in patients with ST-segment elevation and right bundle branch block but structurally normal hearts. Circulation 2000;101:510-5.

15. Lang RM, Bierig M, Devereux RB, et al. Recommendations for chamber quantification: a report from the American Society of Echocardiography's Guidelines and Standards Committee and the Chamber Quantification Writing Group, developed in conjunction with the European Association of Echocardiography, a branch of the European Society of Cardiology. J Am Soc Echocardiogr 2005;18:1440-63.

16. Shewan LG, Coats AJ. Ethics in the authorship and publishing of scientific articles. Int J Cardiol 2010;144:1-2.

17. Dhar Malhotra J, Chen C, Rivolta I, et al. Characterization of sodium channel alphaand beta-subunits in rat and mouse cardiac myocytes. Circulation 2001;103:130310.

18. Schwartz PJ, Moss AJ, Vincent GM, Crampton RS. Diagnostic criteria for the long QT syndrome. An update. Circulation 1993;88:782-4.

19. Junttila MJ, Brugada $P$, Hong $K$, et al. Differences in 12-lead electrocardiogram between symptomatic and asymptomatic Brugada syndrome patients. J Cardiovasc Electrophysiol 2008;19:380-3.

20. Toh N, Morita H, Nagase S, et al. Atrial electrophysiological and structural remodeling in high-risk patients with Brugada syndrome: assessment with electrophysiology and echocardiography. Heart Rhythm 2010;7:218-24.

21. Morita H, Kusano-Fukushima K, Nagase S, et al. Atrial fibrillation and atrial vulnerability in patients with Brugada syndrome. $\mathrm{J}$ Am Coll Cardiol 2002;40:1437-44.

22. Govindan M, Batchvarov VN, Raju H, et al. Utility of high and standard right precordial leads during ajmaline testing for the diagnosis of Brugada syndrome. Heart 2010;96:1904-8. 
